in value was only about $1 \frac{3}{4}$ per-cent. Taking into account, however, the various contingencies that may arise, if the reversion be not converted at the first, the course recommended by the Court seems the most equitable that could be adopted.

$$
\begin{aligned}
& \text { I am, Sir, } \\
& \text { Your obedient Servant, } \\
& \text { J. R. HAR'T, F I.A. }
\end{aligned}
$$

26 St. Andrew Square,

Edinburgh,

8 March 1895.

\title{
MR. RYAN'S PAPER ON VALUATIONS FOR SOLVENCY.
}

To the Editor of the Journal of the Institute of Actuaries.

Srn,-In the hope that they may be of interest, I send herewith tables for endowment assurances payable at age 60, corresponding to those for the whole term of life given in Mr. Ryan's paper recently read before the Institute.

In Mr. Ryan's tables, as well as in my own, I am somewhat surprised to find that the percentage of the reserve values to the premiums paid in certain cases first of all diminishes with the increased duration of the policy and then increases again. The point seems to me worthy of further investigation, and I find that a very simple formula will give the value of $n$ when the percentages cease to diminish or commence to increase.

$$
\text { We have } \quad \frac{{ }_{n} \mathrm{~V}_{x}}{n \mathrm{P}_{x}}>=<\frac{{ }_{n+1} \mathrm{~V}_{x}}{(n+1) \mathrm{P}_{x}} . . . .
$$

according as $(n+1)_{n} \mathrm{~V}_{x}>=<n_{n+1} \mathrm{~V}_{x}$

according as $\frac{{ }_{n} \mathrm{~V}_{x}}{{ }_{n+1} \mathrm{~V}_{x}-{ }_{n} \mathrm{~V}_{x}}>=<n$. . . . . . . . . .

It should be noted that the tables in Mr. Ryan's paper are concerned with the ratios of the successive terms in the two following series:

$$
\begin{aligned}
& n \mathrm{P}_{x},(n+1) \mathrm{P}_{x},(n+2) \mathrm{P}_{x}, \& c . \\
& { }_{n} \mathrm{~V}_{x},{ }_{n+1} \mathrm{~V}_{x},{ }_{n+2} \mathrm{~V}_{x}, \& c .
\end{aligned}
$$

The first of these two series is in arithmetical progression, and its differences are a constant quantity $\left(=P_{x}\right)$. 
The differences in the second series are, however, the results of elements progressing at varying force in different directions, as is evident from the equation

$$
\left({ }_{n} \mathrm{~V}_{x}+\mathrm{P}_{x}\right)(1+i)-q_{x+n}\left(1-{ }_{n+1} \mathrm{~V}_{x}\right)={ }_{n+1} \mathrm{~V}_{x},
$$

whence

$$
\left({ }_{n+1} \mathrm{~V}_{x}-{ }_{n} \mathrm{~V}_{x}\right)=\Delta_{n} \mathrm{~V}_{x}={ }_{n} \mathrm{~V}_{x}(i)+\mathrm{P}_{x}(1+i)-q x+n\left(1-{ }_{n+1} \mathrm{~V}_{x}\right),
$$

where, for all the cases in the tables under consideration,

$$
\left.\begin{array}{l}
{ }_{n} \mathrm{~V}_{x} i \text { increases } \\
\mathrm{P}_{x}(1+i) \text { is constant } \\
\left(1-{ }_{n+i} \mathrm{~V}_{x}\right) \text { decreases } \\
q_{x+n} \text { increases }
\end{array}\right\} \text { as } n \text { increases. }
$$

The net effect of these varying elements in the case of whole term and endowment assurances is to cause the series ${ }_{n} \mathrm{~V}_{x}$, in most tables to increase as $n$ increases, but not at an even rate; and the value of the above expression in (2) must depend on the rates of interest and of mortality used in the calculations.

$$
\text { Your obedient servant, }
$$

28 King Street,

ERNEST WOODS.

Covent Garden, W.C.

\begin{tabular}{|c|c|c|c|c|c|c|c|c|c|c|c|c|}
\hline \multirow{3}{*}{ 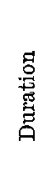 } & \multicolumn{6}{|c|}{$\begin{array}{l}\text { Age at Entri } 25 \text {-OfFich Premiom } \\
\qquad 2.15 \mathrm{~s}, 6 d .\end{array}$} & \multicolumn{6}{|c|}{$\begin{array}{l}\text { AGe at Entry } 35-\text { OfFice PRemium } \\
\qquad 4.3 s .0 d .\end{array}$} \\
\hline & \multirow{2}{*}{$\mathrm{V}(\mathrm{I})$} & \multirow{2}{*}{$\mathrm{V}(2)$} & \multirow{2}{*}{$\mathrm{V}(3)$} & \multicolumn{3}{|c|}{$\begin{array}{l}\text { Percentage to } \\
\text { Prems. paid of }\end{array}$} & \multirow{2}{*}{$\mathrm{V}(1)$} & \multirow{2}{*}{$\mathrm{V}(2)$} & \multirow{2}{*}{$\mathrm{V}(3)$} & \multicolumn{3}{|c|}{$\begin{array}{l}\text { Percentage to } \\
\text { Prems. pald of }\end{array}$} \\
\hline & & & & $V(1)$ & $\mathrm{V}(2)$ & $\mathrm{V}(3)$ & & & & $\mathrm{V}(1)$ & $\mathrm{V}(2)$ & $\mathrm{V}(3)$ \\
\hline 3 & -1.9 & $-\quad 7$ & +.5 & & & 6 & $1 \cdot 3$ & $2 \cdot 8$ & $4: 2$ & 11 & 22 & \\
\hline 5 & $+1 \cdot 7$ & $+2 \cdot 8$ & 4.0 & 12 & 20 & 29 & $7 \cdot 2$ & 8.5 & $9 \cdot 9$ & 35 & 41 & $\begin{array}{l}3 \\
48\end{array}$ \\
\hline 10 & $11 \cdot 5$ & $12 \cdot 6$ & $13 \cdot 6$ & 42 & 45 & 49 & $24: 0$ & $25 \cdot 1$ & $26 \cdot 2$ & 58 & 61 & 63 \\
\hline 20 & $37 \cdot 1$ & $37 \cdot 8$ & 38.5 & 67 & 68 & 69 & $68 \cdot 6$ & $69 \cdot 1$ & $69 \cdot 5$ & 83 & 83 & 84 \\
\hline 30 & $74: 0$ & $74: 3$ & $74 \cdot 6$ & 89 & 89 & 90 & .. & & & $\cdots$ & $\ldots$ & $\cdots$ \\
\hline
\end{tabular}

30 March 1895.

Valtations for Solvency.

Endowment Assurances payable at 60 or Death.

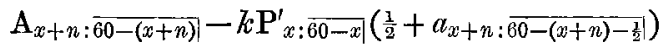

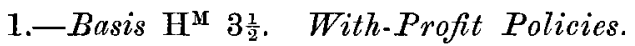


Valdations for Solvency--(continued).

Endowment Assurances payable at 60 or Death-(continued).

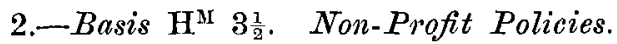

\begin{tabular}{|c|c|c|c|c|c|c|c|c|c|c|c|c|}
\hline \multirow{3}{*}{ 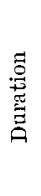 } & \multicolumn{6}{|c|}{$\begin{array}{l}\text { AGE at ENTRy 25-OFfice Premiom } \\
\& 2.8 s .10 \mathrm{~d} \text {. }\end{array}$} & \multicolumn{6}{|c|}{$\begin{array}{c}\text { Age at Extry } 35-\text { OFITCE Premium } \\
\& 3.13 s \text { od. }\end{array}$} \\
\hline & \multirow{2}{*}{$\mathrm{V}(1)$} & \multirow{2}{*}{$V(2)$} & \multirow{2}{*}{$\mathrm{V}(3)$} & \multicolumn{3}{|c|}{$\begin{array}{l}\text { Percentage to } \\
\text { Prems. paid of }\end{array}$} & \multirow{2}{*}{$\mathrm{V}(1)$} & \multirow{2}{*}{$\mathrm{V}(2)$} & \multirow{2}{*}{$V(3)$} & \multicolumn{3}{|c|}{$\begin{array}{l}\text { Percentage to } \\
\text { Prems. paid of }\end{array}$} \\
\hline & & & & $\mathrm{V}(1)$ & $\mathrm{v}(2)$ & $v(3)$ & & & & $\mathrm{V}(1)$ & $\mathrm{V}(2)$ & $\mathrm{v}(3)$ \\
\hline 3 & $3 \cdot 3$ & $4 \cdot 3$ & $5 \cdot 3$ & 44 & 59 & 73 & $7 \cdot 5$ & $8 \cdot 7$ & $10^{\circ} 0$ & 68 & 80 & 91 \\
\hline 5 & 6.6 & $7 \cdot 6$ & $8 \cdot 6$ & 54 & 62 & 71 & $13 \cdot 0$ & $14 \cdot 2$ & $15 \cdot 4$ & 71 & 78 & 84 \\
\hline 10 & $16 \cdot 0$ & 16.9 & $17 \cdot 8$ & 65 & 69 & 73 & $28 \cdot 8$ & $29 \cdot 7$ & $30 \cdot 7$ & 79 & 82 & 84 \\
\hline 20 & $40 \cdot 2$ & 40.9 & 41.5 & 82 & 84 & 85 & $70 \cdot 6$ & $71 \cdot 0$ & $71 \cdot 4$ & 97 & 97 & 98 \\
\hline 30 & $75 \cdot 3$ & $75 \cdot 6$ & $75 \cdot 8$ & 103 & 103 & 104 & $\cdots$ & . & & $\ldots$ & r. & $\ldots$ \\
\hline
\end{tabular}

3.-Basis $\mathrm{H}^{\mathrm{M}}$ 4. With-Profit Policies.

\begin{tabular}{|c|c|c|c|c|c|c|c|c|c|c|c|c|}
\hline \multirow{3}{*}{ 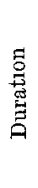 } & \multicolumn{6}{|c|}{$\begin{array}{c}\text { Age at Entry } 25 \text {-OFfice Premiom } \\
£ 2.15 s .6 d .\end{array}$} & \multicolumn{6}{|c|}{$\begin{array}{c}\text { Age at Entry } 35-\text { OfFicl Premidm } \\
£ 4.3 s .0 d .\end{array}$} \\
\hline & \multirow{2}{*}{$\mathrm{V}(1)$} & \multirow{2}{*}{$\mathrm{V}(2)$} & \multirow{2}{*}{$\mathrm{V}(3)$} & \multicolumn{3}{|c|}{$\begin{array}{l}\text { Percentage to } \\
\text { Prems. pard of }\end{array}$} & \multirow{2}{*}{$\mathrm{V}(1)$} & \multirow{2}{*}{$\mathrm{V}(2)$} & \multirow{2}{*}{$V(3)$} & \multicolumn{3}{|c|}{$\begin{array}{l}\text { Percentage to } \\
\text { Prems. paid of }\end{array}$} \\
\hline & & & & $\mathrm{V}(1)$ & $V(2)$ & $\mathrm{V}(3)$ & & & & $\mathrm{V}(1)$ & $\mathrm{V}(2)$ & $\mathrm{V}(3)$ \\
\hline 3 & - $4 \cdot 0$ & $-2 \cdot 8$ & $-1 \cdot 7$ & & $\cdots$ & & $-\quad .9$ & +5 & $+1 \cdot 8$ & & 4 & 15 \\
\hline 5 & $-\quad \cdot 6$ & $+\cdot 5$ & $+1 \cdot 6$ & & 3 & 11 & +48 & $6 \cdot 1$ & $7 \cdot 4$ & 23 & 29 & 36 \\
\hline 10 & $+8 \cdot 8$ & $9 \cdot 8$ & $10 \cdot 8$ & 32 & 35 & 39 & $21 \cdot 4$ & $22 \cdot 5$ & 235 & 52 & 54 & 57 \\
\hline 20 & $34 \cdot 0$ & $34 \cdot 8$ & $35 \cdot 5$ & 61 & 63 & 64 & 66.9 & $67 \cdot 3$ & 67.8 & 81 & 81 & 82 \\
\hline 30 & $72 \cdot 2$ & $72 \cdot 5$ & $72 \cdot 8$ & 87 & 87 & 87 & $\ldots$ & $\ldots$ & $\cdots$ & $\ldots$ & $\ldots$ & $\ldots$ \\
\hline
\end{tabular}

4.-Basis $\mathrm{H}^{\mathrm{M}}$ 4. Non-Profit Policies.

\begin{tabular}{|c|c|c|c|c|c|c|c|c|c|c|c|c|}
\hline \multirow{3}{*}{ 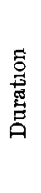 } & \multicolumn{6}{|c|}{$\begin{array}{c}\text { Age at Entry } 25 \text {-OfFice Premium } \\
\qquad 2.8 s .10 d .\end{array}$} & \multicolumn{6}{|c|}{$\begin{array}{c}\text { Age at EnTRX } 35-\text { OfFICE PREMIOM } \\
\qquad 3,13 s \text {. 0d. }\end{array}$} \\
\hline & \multirow{2}{*}{$\mathrm{V}(1)$} & \multirow{2}{*}{$\mathrm{V}(2)$} & \multirow{2}{*}{$\mathrm{V}(3)$} & \multicolumn{3}{|c|}{$\begin{array}{l}\text { Percentage to } \\
\text { Prems. pard of }\end{array}$} & \multirow{2}{*}{$\mathrm{V}(\mathrm{I})$} & \multirow{2}{*}{$\mathrm{V}(2)$} & \multirow{2}{*}{$\mathrm{V}(3)$} & \multicolumn{3}{|c|}{$\begin{array}{l}\text { Percentage to } \\
\text { Prems paid of }\end{array}$} \\
\hline & & & & $\mathrm{V}(1)$ & $\mathrm{V}(2)$ & $\mathrm{V}(3)$ & & & & $\mathrm{V}(1)$ & $\mathrm{v}(2)$ & $\mathrm{V}(3)$ \\
\hline 3 & .9 & 1.9 & $2 \cdot 9$ & 12 & 25 & 39 & $5 \cdot 0$ & $6 \cdot 2$ & $7 \cdot 4$ & 46 & 57 & 68 \\
\hline 5 & $4 \cdot 1$ & $5 \cdot 0$ & $6 \cdot 0$ & 33 & 41 & 49 & $10 \cdot 4$ & $11 \cdot 5$ & $12 \cdot 7$ & 57 & 63 & 69 \\
\hline 10 & $13 \cdot 1$ & 13.9 & $14 \cdot 8$ & 53 & 57 & 61 & $26^{\circ} 0$ & $26 \cdot 9$ & $27 \cdot 9$ & 71 & 74 & 76 \\
\hline 20 & $37 \cdot 1$ & $37 \cdot 7$ & $38 \cdot 4$ & 76 & 77 & 79 & $68 \cdot 8$ & $69 \cdot 2$ & $69 \cdot 6$ & 94 & 95 & 95 \\
\hline 30 & 73.5 & $73 \cdot 8$ & $74: 0$ & 100 & 101 & 101 & $\cdots$ &. & $\cdots$ & 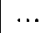 & & $\ldots$ \\
\hline
\end{tabular}

